\title{
Nouvelles directives pour le don d'organes solides par des personnes vivantes
}

Jürg Steiger ${ }^{a}$, Michelle Salathéb

a Prof., président de la souscommission

b lic. en droit, secrétaire générale adjointe de l'ASSM
Correspondance:

Michelle Salathé, lic. en droit ASSM

Petersplatz 13

CH-4051 Bâle

Tel. 0612699030

Fax 0612699039

mail@samw.ch

www.samw.ch
De nos jours, la transplantation d'organes représente une forme de thérapie prometteuse dont l'efficacité n'est plus à prouver. Les progrès dans le domaine des soins médicaux et chirurgicaux ont augmenté l'espérance de vie des receveurs d’organes et amélioré leur qualité de vie. Les besoins d'organes sont, de ce fait, en augmentation constante. Alors même que le nombre de dons d'organes provenant de donneurs décédés a tendance à diminuer, la disposition au don d'organes par des personnes vivantes est plus élevée en Suisse. Si le don d'organes par des donneurs vivants gagne en importance, c'est, d'une part, à cause des longues listes d'attente et, d'autre part, parce que les chances de succès sont nettement supérieures. Dans ce contexte, le Sénat de l'Académie Suisse des Sciences Médicales (ASSM) a adopté le 20 mai 2008 les nouvelles directives médico-éthiques «Don d'organes solides par des personnes vivantes».

La nouvelle Loi fédérale sur la transplantation d'organes et de tissus et les ordonnances correspondantes, entrées en vigueur le $1^{\text {er }}$ juillet 2007, offrent une réglementation détaillée de ce domaine complexe de la médecine. C'est pour cette raison que les nouvelles directives contiennent divers renvois à la loi sur la transplantation et aux ordonnances correspondantes. Ces directives, qui se limitent au don d'organes solides par des personnes vivantes (notamment de foies et de reins), ne sont pas destinées à combler un vide juridique, mais à apporter une aide à la mise en pratique des dispositions légales. Elles s'adressent aux médecins, soignants et autres professionnels de la santé impliqués directement dans l'évaluation des donneurs potentiels.

La législation part du principe que chaque personne majeure et capable de discernement a le droit de décider de façon libre et responsable, après avoir été informée en détail, si elle veut courir le risque lié à un don d'organe en faveur d'une tierce personne. Les directives précisent que les médecins concernés doivent respecter la décision du donneur si elle est prise de façon autonome, mais qu'ils ont, en même temps, le devoir de ne pas nuire au donneur. Toutefois, la protection du donneur doit toujours constituer une priorité. Lorsque les risques pour la santé et la vie du donneur sont trop élevés, le don d'organe doit être refusé, même si ce refus est contraire à la volonté du donneur. Dans chaque cas particulier, de nombreux facteurs interviennent dans la pesée des risques et de l'utilité. Pour assurer la protection des donneurs, il convient non seulement de tenir compte des aspects médicaux, mais également des facteurs psychosociaux, tels que les conséquences psychiques d'un don d'organe respectivement du refus d'un don. Un don d'organe doit également être refusé lorsqu'il s'avère que la décision résulte d'une pression extérieure.

Lors de la consultation, la première version des directives a été accueillie très positivement, mais quelquefois aussi remise en question de façon critique. La sous-commission, dirigée par le Prof. Jürg Steiger de Bâle, a remanié le texte des directives en tenant compte des suggestions et remarques exprimées pendant la période de consultation. Elles comprennent ainsi un nouveau chapitre concernant le libre consentement et un autre concernant les donneurs qui refusent la transfusion sanguine; par ailleurs, la souscommission a exposé plus précisément les réflexions éthiques à la base du don d'organes par des personnes vivantes. De même, les directives prévoient à présent qu'en cas de doute quant à l'aptitude au don, un avis externe peut être recherché - tout en garantissant le respect des droits de la personne du couple donneur-receveur.

Avec les directives «Don d'organes solides par des personnes vivantes», l'ASSM veut apporter son soutien aux médecins, soignants et autres professionnels de la santé impliqués dans l'évaluation médicale et psychosociale des donneurs et dans leur suivi. Comme la plupart des directives de l'ASSM, celles-ci contiennent de nombreuses recommandations adressées aux centres de transplantations, à la Conférence des directeurs de la santé, au législateur et aux assureurs qui devraient contribuer à la mise en pratique de ces directives.

Les directives peuvent être téléchargées sur le site internet de I'ASSM (www.assm.ch $\rightarrow$ ÉTHIQUE) ou commandées gratuitement, sous forme de brochure, au secrétariat général de I'ASSM, Petersplatz 13, 4051 Bâle. 